\title{
To kinetic modeling of solar wind over magnetoactive regions and beyond
}

\author{
V. M. Gubchenko ${ }^{1}$, V. V. Zaitsev ${ }^{1}$, \\ H.K. Biernat ${ }^{2}$, M.L. Khodachenko ${ }^{2}$, and H. O. Rucker ${ }^{2}$ \\ ${ }^{1}$ Institute of Applied Physics, Russian Academy of Science, Nizhny Novgorod, email: \\ ua3thw@appl.sci-nnov.ru \\ ${ }^{2}$ Space Research Institute, Austrian Academy of Science, Graz, email: \\ maxim.khodachenko@oeaw.ac.at
}

\begin{abstract}
We model hot collisionless solar corona/slow solar wind (SW) by kinetic Vlasov equation. The equatorial region with an unprescribed magnetic field is under electromagnetic action, and we have formation of a 3D Quasi-Stationary Coronal Structures (QSCS) in the current-carrying (CC) plasma with SW flows. The QSCS are generated via electromagnetic mode development in plasma having anisotropic velocity distribution function (VDF). As a result we get streamers like stratification mode, CMEs like tearing mode and heliospheric sheet like pinch mode in anisotropic plasma. The scales in the QSCS are defined via angle dependent magnetic Debye scale. The scale itself depends on value and sign of plasma anisotropy parameter which is calculated for postulated plasma VDF.
\end{abstract}

The QSCSs are associated with Current Carrying (CC) plasma of a Heliospheric Current Sheet (CS) and slow SW. "Closed" magnetic field lines in the QSCSs with magnetic islands are associated with Coronal Mass Ejections (CMEs), whereas "ray" structures above, are associated with magnetic flux tubes and streamers forming a streamer belt in the azimuthal direction.

Within the kinetic approach we have in the polar regions of the Sun a "parallel" expansion of a non-Current-Carrying (non-CC) coronal plasma characterized by the concentration $n_{\alpha}$ and temperature $T_{\alpha}$. Strongly magnetized charged particles move there along the quasi-radial prescribed magnetic field lines $\mathbf{B}=\nabla \times \mathbf{A} \approx \mathbf{B}_{r}$ and form under action of the electrostatic field $\left(\mathbf{E}_{l}=-\nabla \varphi\right)$ a high speed solar wind. At the ecliptic plane, above the magnetoactive regions on the solar surface, we have a "perpendicular" expansion of a CC plasma, where particles are weakly magnetized and move perpendicular to the unprescribed equatorial magnetic field. The equatorial/ecliptic region has a more complicated physics behind. We have there a CC plasma of the heliospheric CS structured by the magnetic field into the QSCSs. The magnetic field $\mathbf{B} \approx \mathbf{B}_{n}$ normal to the ecliptic plane is selfconsistent with the radial flows of the slow solar wind plasma and its double humped VDF. Thus it can not be prescribed in value and topology as it has been done in the polar regions. Expansion of the corona in the equatorial/ecliptic region is accompanied by the eddy plasma motions and excitation of Transverse Electromagnetic (TEM) fields $\mathbf{E}_{t}=-c^{-1} \partial \mathbf{A} / \partial t$. Due to high temperature and slowness of the SW the QSCSs here are characterized by Landau type collisionless dissipation (relaxation) appeared via acceleration of some charged particles on the VDF by TEM fields forming resistive current $\mathbf{j}_{r}$. As a result we get magnetic reconnection effect transforming magnetic field topology formed by initial diamagnetic current $\mathbf{j}_{d}$ into observed QSCSs. We suppose that the plasma is close to the diamagnetic state, i.e. $\mathbf{j}_{r}<<\mathbf{j}_{d}$.

We postulate that plasma in the diamagnetic CC state has a double humped VDF. It means that plasma consists of two components and is parametrized additionally by two 
values for concentration $n_{\alpha}=n_{c}+n_{w}$ and by values for flows producing azimuthal current $u_{\alpha}$ and double flows in radial direction $\Delta u=u_{w}-u_{c} \neq 0$ describing SW expansion. The model of the diamagnetic VDF for this state is $f_{d \alpha}(\mathbf{v}, x)=f_{c \alpha}+f_{w \alpha}$, where

$$
\begin{gathered}
f_{c \alpha}=\frac{n_{c 0}}{\left(2 \pi v_{\alpha}\right)^{3 / 2}} \exp \left[-\frac{\left.v_{x}^{2}+\left(v_{y}-u_{\alpha}\right)^{2}+\left(v_{z}-u_{c}\right)^{2}\right)}{2 v_{\alpha}^{2}}\right], \\
f_{w \alpha}=\frac{n_{w}}{\left(2 \pi v_{\alpha}\right)^{3 / 2}} \exp \left[-\frac{\left.v_{x}^{2}+v_{y}^{2}+\left(v_{z}-u_{w}\right)^{2}\right)}{2 v_{\alpha}^{2}}\right] .
\end{gathered}
$$

Here $v_{\alpha}^{2}=\left(T_{\alpha} / m_{\alpha}\right)(\alpha=e, i)$ is the thermal velocity with temperature $T_{\alpha} ; m_{\alpha}$ is the mass of particles.

We can get an idea on the QCS formation, its dynamic and spatial scales studying more realistic dissipative quasi-diamagnetic state of the VDF $f_{\alpha}(\mathbf{v}, x)=f_{d \alpha}+f_{r \alpha}$. selfconsistent with excitation via Weibel instability of 3D TEM fields. The TEM mode producing heliospheric CS structure with $\mathbf{B} \approx \mathbf{B}_{r}(x)$ has wave number perpendicular to ecliptic plane $\mathbf{k}=k_{x} \mathbf{x}_{0}, \mathbf{A}=A_{y} \mathbf{y}_{0}$. The TEM modes, producing $\mathbf{B}_{n}$ in the ecliptic plane in the neutral sheet, can appear in two forms. First, it can be a tearing mode with $\mathbf{k}=k_{z} \mathbf{z}_{0}$ along radial direction, $\mathbf{A}=A_{y} \mathbf{y}_{0}$, which forms closed and open structures with transients $(r, \theta)$. And second, it can be a stratification (kink, sausage) mode with $\mathbf{k}=k_{y} \mathbf{y}_{0}$ along asimuthal direction, $\mathbf{A}=A_{z} \mathbf{z}_{0}$, which forms the ray belt structures, $(r, \phi)$ in the corona.

A characteristic spatial inhomogeneity scale of fine QSCS in a $3 \mathrm{D}$ corona is $L \approx$ $r_{D M}(\mathbf{k} /|\mathbf{k}|)$. The scale originated from the range of TEM mode instability $0<|\mathbf{k}| r_{D M}<$ 1 in CC plasma with flows. And it has sense to speak about the range $0<|\mathbf{k}| r_{D M}<1$ of the Weibel instability only when $r_{D M}^{2}>0$. The $r_{D M}$ is magnetic Debye scale for the CC plasma with flows $r_{D M}^{-2}(\mathbf{k} /|\mathbf{k}|)=\Sigma_{\alpha} \kappa_{\alpha}(\mathbf{k} /|\mathbf{k}|) \omega_{p \alpha}^{2} / c^{2}$, here $\omega_{p \alpha}^{2}=4 \pi e^{2} n_{c 0} / m_{\alpha}$ is the plasma frequency and $c$ is the speed of light.

As an important characteristic of the considered problem appears the plasma anisotropy parameter $\kappa_{\alpha}$. Its form is angle dependent on the wave vector direction characterizing the considered electromagnetic structure and, in general depends on the VDF parameters: currents $u_{\alpha}$ and plasma flows $\left(u_{w}, u_{c}\right)$. For the heliospheric CS formation TEM mode, having an $x$-dependent structure and $\mathbf{k}=k_{x} \mathbf{x}_{0}$, the plasma anisotropy parameter is $\kappa_{\alpha}=\kappa_{c \alpha}=u_{\alpha}^{2} / v_{\alpha}^{2}$ and produce Harris sheet scale $L=L_{H}$. Its value does not depend on flows, and is essentially positive. For the cases of $\mathbf{k}=k_{z} \mathbf{z}_{0}$ and $\mathbf{k}=k_{y} \mathbf{y}_{0}$, the sign and value of $\kappa_{\alpha}$ depend not only on the current but also on flows. From linear analysis we can get that $\kappa_{y \alpha}=\kappa_{c \alpha}-\kappa_{\alpha w}=\kappa_{c \alpha}-\Delta M_{e}^{2} \beta_{w} /\left(\beta_{w}+1\right)$, where $\Delta M_{\alpha}=\Delta u / v_{\alpha}, \beta_{w}=n_{w} / n_{c}$. The scale $r_{D M y}$ of the magnetic islands along $\mathbf{z}_{0}$ can be found from $r_{D M y}^{-2}=\sum_{\alpha} \kappa_{y \alpha}\left(\omega_{p c \alpha}^{2} / c^{2}\right)$. The scale of magnetic ropes along $\mathbf{y}_{0}$ is defined by $r_{D M z}^{-2}=\sum_{\alpha} \kappa_{z \alpha}\left(\omega_{p w \alpha}^{2} / c^{2}\right)$. Finally we obtain $n_{c} \kappa_{y e}+n_{w} \kappa_{z e} \approx 0$. Thus, assuming the radial dependence of parameters $\kappa_{y}(r)$ and $\kappa_{z}(r)$, in accordance with observed picture of the QSCS we obtain that at distances $r<r_{n}$ the mode $A_{y}$ is unstable, whereas the mode $A_{z}$ is stable, and vice versa at $r>r_{n}$.

\section{Acknowledgements}

This work has been supported by RFBR grants 02-02-16239, 02-02-04005, 03-02-2009, by LSS grant 1744.2003 .2 , by Presidium and Division of Physics of the RAS, by the Austrian Academy of Sciences (Academic Research Collaboration Programme) and by FWF projects P17100-N08, P16919-N08. 